\title{
Tumor-Derived Extracellular Vesicles: Their Role in Immune Cells and Immunotherapy
}

\author{
Qi $\mathrm{Li}^{1}$ \\ Suna Cai ${ }^{1}$ \\ Mengjiao $\mathrm{Li}^{1}$ \\ Kab Ibrahim Salma' \\ Xiaojie Zhou' \\ Feiyu $\mathrm{Han}^{2}$ \\ Jinzhao Chen ${ }^{2}$ \\ Ting Huyan ${ }^{1,3}$
}

'Key Laboratory for Space Biosciences and Biotechnology, Institute of Special Environment Biophysics, School of Life Sciences, Northwestern Polytechnical University, Xi'an, 7I0072, People's Republic of China; ${ }^{2}$ Shanxi

Weiqidaguangming Pharmaceutical Co., Ltd, The First Medical Park, Economic \& Technology Development Zone, Datong, 037000, People's Republic of China; ${ }^{3}$ Institute of Flexible Electronics (IFE), Northwestern Polytechnical University, Xi'an, 710072, People's Republic of China

Correspondence: Ting Huyan Key Laboratory for Space Biosciences and Biotechnology, Institute of Special Environment Biophysics, School of Life Sciences, Northwestern Polytechnical University, Xi'an, 710072, People's

Republic of China

$\mathrm{Tel} / \mathrm{Fax}+862988460332$

Email huyanting@nwpu.edu.cn

\begin{abstract}
Nowadays, tumor has been the serious threat to human health and life. To further explore the mechanism of tumor genesis and development is necessarily for developing the effective treatment strategy. Extracellular vesicles are the vesicles secreted by almost all types of cells, and they play an important part in intercellular communication by transporting their cargoes. Immune cells are the vital components of the human defense system, which defense against infection and tumor through cytotoxicity, immune surveillance, and clearance. However, via release tumor-derived extracellular vesicles, tumor could induce immune cells dysfunction to facilitate its proliferation and metastasis. Studies have shown that tumorderived extracellular vesicles play dual role on immune cells by their specific cargoes. Here, we reviewed the effects of tumor-derived extracellular vesicles on immune cells in recent years and also summarized their research progress in the tumor immunotherapy and diagnosis.
\end{abstract}

Keywords: tumor-derived extracellular vesicles, immune cell, regulatory effects, drug delivery, immunotherapy

\section{Introduction}

Extracellular vesicles (EVs) are a class of plasma membrane encapsulated, cellsecreted vesicles with a diameter ranging from 30 to $1500 \mathrm{~nm} .^{1}$ EVs are mainly classified into two subtypes by size: ${ }^{2}$ (1) medium/large EVs, with diameters of 150 $1500 \mathrm{~nm}$, including microvesicles, also called ectosomes, and apoptotic bodies, which are formed by cell membrane budding or apoptosis; and (2) small EVs, with diameters from 30 to $150 \mathrm{~nm}$, including exomeres and exosomes. Exosomes are formed through exocytosis after the plasma membrane fuses with multivesicular bodies (MVBs), which are formed by the budding of early endosomes via the invagination of the cell membrane. ${ }^{3,4}$ We reviewed the role of tumor-derived extracellular vesicles (TEVs) from the existing literature, mainly focusing on exosomes, immune cells and tumor immunotherapy. The extracellular vesicles, exosomes or microvesicles covered in the original articles are discussed in the text.

Exosomes are enriched in tetraspanins, including CD9, CD63, CD81, and CD82, which are involved in exosome formation and are considered classical exosomal markers. NSMASE2 (also known as SMPD3) and RAB27A are the two critical enzymes for exosomes biogenesis, the former promotes vesicle budding from the early endosome, while the latter is involved in the fusion of multivesicular bodies with cell membrane. ${ }^{5}$ Secreted by local cells and tissues, exosomes can be transported to all over the body through the circulatory system to play a remote 
regulatory role. ${ }^{6}$ Furthermore, exosomes carry many proteins, lipids, and nucleic acids and have a similar topological structure to the cell of origin; thus, they reflect the characteristics of the original cells to some extent. ${ }^{7}$ For example, tumor-derived exosomes (TEXs) generated from different types of tumors carry specific "molecular cargoes" that mainly originate from the cell membrane and endosomes of the original tumor cells. ${ }^{5}$ These TEXs have enormous potential for use as specific biomarkers in the early screening, diagnosis, and prognosis of cancer in the clinic. $^{8}$

The tumor microenvironment (TME) is the inner environment involved in tumor generation and development and is composed of malignant cells, cancer-associated fibroblasts, neuroendocrine cells, adipose cells, immunocytes, and vascular and lymphatic networks. ${ }^{9}$ If the primary tumor is to grow in secondary organs, its first task is to evade immune clearance. ${ }^{10} \mathrm{~A}$ previous study revealed that the released amount of TEXs is 10 -fold greater than that of normal cell-derived exosomes. ${ }^{11}$ In the TME, tumors secrete a large number of TEVs to induce immune cell dysfunction and activate immune suppressor cells to impair antitumor immune surveillance, promote TME formation and assist tumors in escaping from the host immune system. ${ }^{12}$ Based on the existing literature, in this article, we reviewed the regulatory effects of TEVs on immune cells in vitro and in vivo and the potential for using 1) TEVs as biomarkers for cancer diagnoses; 2) TEVs as vehicles to load drugs for cancer therapy; and 3) TEVs as vaccines in antitumor immunotherapy.

\section{The Regulatory Effect of TEVs on Immune Cells \\ T Lymphocyte}

$\mathrm{T}$ cells, which are thymus-dependent lymphocytes, have important biological functions of responding to specific antigens, directly killing target cells, and secreting cytokines, ${ }^{13}$ thus playing an essential role in antiinfection and antitumor processes.

\section{TEVs Inhibit Activation and Proliferation and Promote the Apoptosis of T Cells}

Under normal circumstances, through a variety of positive and negative regulatory procedures, the human body can control the intensity of the immune response in a timely manner to maintain the balance and stability of immune function. ${ }^{14} \mathrm{~A}$ large number of studies have revealed that TEVs can take advantage of the self-negative feedback regulation mechanism to create a microenvironment that is conducive to tumor growth. Several cytokines and factors, such as prostaglandin E2, programmed death ligand-1 (PD-L1), IL-10, transforming growth factor- $\beta$ (TGF- $\beta$ ) and adenosine, play important roles in different stages of the immune response ${ }^{15}$ and can be used by TEVs to inhibit $\mathrm{T}$ cell activation and proliferation. ${ }^{16}$ For instance, mediated by CD39 and CD73, exosomes derived from diverse cancer cells can initiate an exogenous nucleotidase cascade by degrading ATP to increase the extracellular adenosine level. Then, extracellular adenosine can inhibit $T$ cell activation by binding to $T$ cell surface receptors (A1, $\mathrm{A} 2 \mathrm{~A}, \mathrm{~A} 2 \mathrm{~B}, \mathrm{~A} 3)$ and reduce the local immune response. ${ }^{17}$ Furthermore, studies have confirmed that most TEVs carrying PD-L1 can bind to PD-1 expressed on activated $\mathrm{T}$ cells to suppress $\mathrm{T}$ cell receptor (TCR)-related pathways and trigger immunosuppressive effects on $\mathrm{T}$ cells. ${ }^{18,19}$ Subsequently, inhibitors that can block the interaction between PD-1 and PD-L1 have been developed to restore the killer function of immune cells. ${ }^{20,21}$ Fas (CD95), also known as death receptor, is a transmembrane receptor existing on the cell membrane. When TEXs carrying Fas ligand (FasL) bind to Fas on the T cell surface, the transmembrane transmission of apoptosis signals is initiated and subsequently induces $\mathrm{T}$ cell apoptosis. ${ }^{22}$ In addition, several in vitro experiments have shown that TEXs isolated from ascites and other malignant effusions from tumor patients carry FasL, which suppresses the activity of effector $\mathrm{T}$ cells by combining with Fas expressed on $\mathrm{T}_{\text {cells }}{ }^{23}$ (Figure 1).

\section{TEVs Cause T Cell Dysfunction}

MicroRNAs (miRNAs), a type of endogenous noncoding RNA containing 19-24 nucleotides, can exert regulatory functions in cells by binding to the 3'UTR or open reading frame of target mRNA and cause target mRNA silencing. ${ }^{24}$ Through encapsulation in exosomes or microvesicles, tumor-derived miRNAs can spread around the TME and even to other parts of the body, where they affect distal cells, including immune cells. The regulation of tumorderived exosomal miRNAs on immune cells is reviewed in Table 1. One study ${ }^{25}$ demonstrated that compared with NP69 cells (an immortalized human nasopharyngeal epithelium cell line) and healthy donor sera, miR-24-3P was significantly overexpressed in exosomes derived from nasopharyngeal carcinoma (NPC) cell lines and patient sera. This miRNA can directly prevent $\mathrm{T}$ cell function by targeting fibroblast growth factor 11 (FGF11). Additionally, Ye 


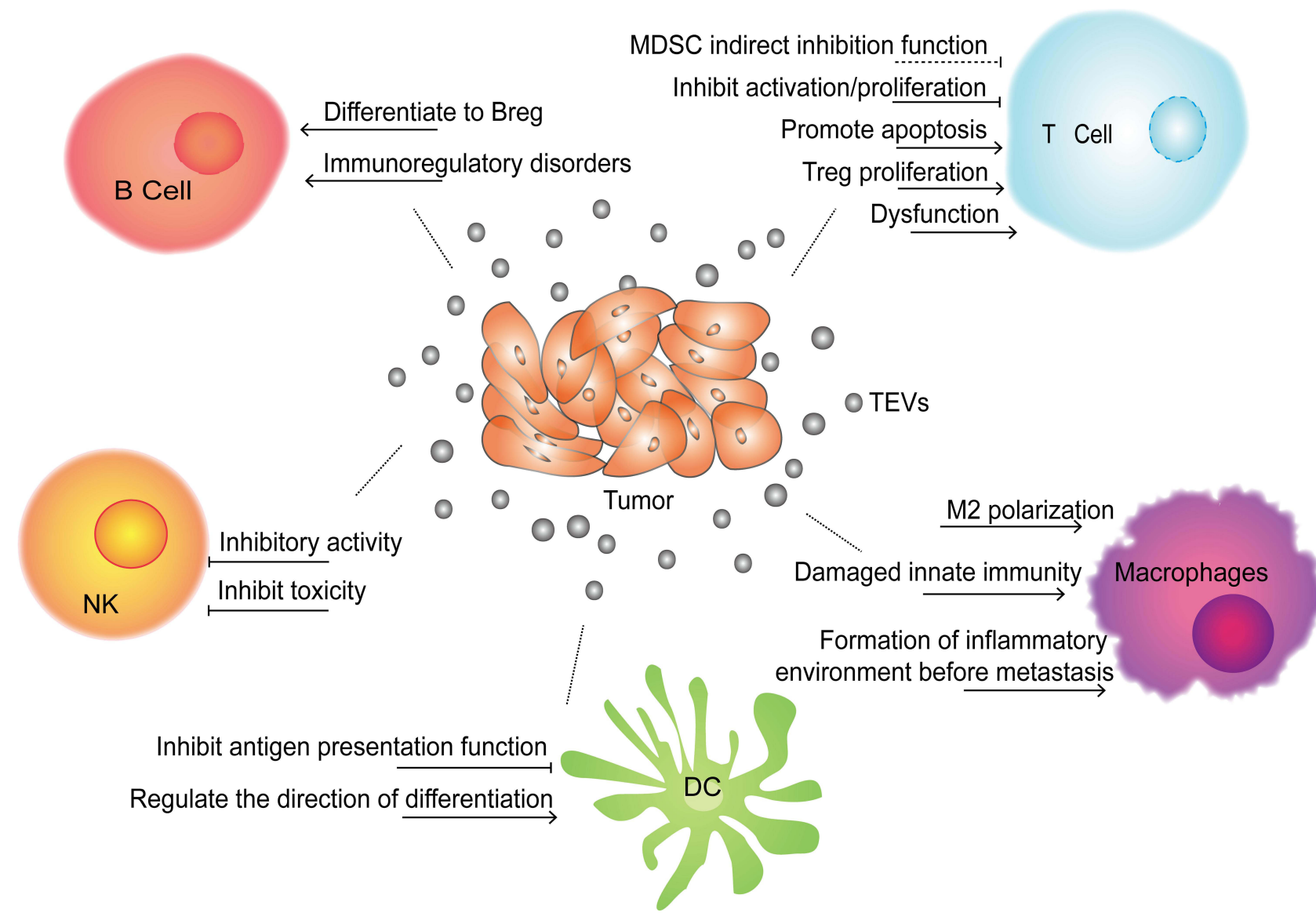

Figure I Effects of TEVs on immune cells. TEVs can directly or indirectly inhibit the functions of immune cells (T cell, B cell, DC, macrophages and NK cell) through a variety of ways, and help tumor escape immune surveillance and clearance, thereby promoting tumor occurrence, growth and metastasis.

et al found ${ }^{26}$ that a group of miRNAs, including miR-106a5p, miR-24-3p, miR-1908, miR-20a-5p, and miR-891a, enveloped in nasopharyngeal carcinoma TW03 cellderived exosomes could induce $\mathrm{T}$ cell dysfunction by downregulating the expression of MAPK1 and blocking the JAK/ STAT pathway. In addition regulating immunocytes via exosomal nucleic acids, TEX-carrying proteins can inhibit $\mathrm{T}$ cell activity. $14-3-3 \zeta$ is a protein that is overexpressed in hepatocellular carcinoma (HCC), which promotes cell proliferation and epithelial-mesenchymal transition (EMT) in HCC. ${ }^{27}$ It was shown that when carried by HCC exosomes, the $14-3-3 \zeta$ protein can be transmitted to tumor infiltrating $\mathrm{T}$ lymphocytes and inhibit their antitumor function. Another study ${ }^{28}$ found that functional PD-L1 carried by breast cancer-derived exosomes can be transferred to other low- or non-expressing PD-L1 tumor cells to shape the TME and inhibit $\mathrm{T}$ cell function. Moreover, a recent study showed that under endoplasmic reticulum (ER) stress, exosomal miR-23a-3p in HCC cells can enhance the expression of PD-L1 in macrophages via the phosphatase and tensin homolog (PTEN)-phosphatidylinositol 3-kinase-protein kinase $\mathrm{B}$ (AKT) pathway to inhibit $\mathrm{T}$ cell function. ${ }^{29}$ Additionally, it was reported that interferon- $\gamma$ (IFN- $\gamma$ ) can increase the expression of PD-L1 on human melanomaderived exosomes, which further inhibits the function of $\mathrm{CD}^{+}$T cells. $^{30}$

\section{TEVs Promote Treg Proliferation}

Regulatory $\mathrm{T}$ cells (Tregs) are a particular subgroup of $\mathrm{T}$ cells that play a negative regulatory role in the immune response and are important for maintaining immune tolerance in the human body. By secreting inhibitory factors such as TGF- $\beta$ and IL-10, Tregs can inhibit $\mathrm{CD}^{+} / \mathrm{CD} 8^{+}$ $\mathrm{T}$ cell activity and promote tumor growth in the TME. ${ }^{31}$ Colorectal EVs are able to induce $\mathrm{T}$ cell differentiation into Treg cells by activating TGF- $\beta /$ SMAD signaling and inactivating SAPK signaling. ${ }^{32}$ Other studies have found that by inhibiting the ERK, STAT1, and STAT3 pathways and enhancing the phosphorylation level of STAT5, nasopharyngeal carcinoma exosomes can induce an increase in $\mathrm{CD}^{+} / \mathrm{CD}^{+} \mathrm{T}$ cell secretion of the proinflammatory 
Table I The Inhibitory Effect of TEVs on Immune Cells

\begin{tabular}{|c|c|c|c|c|}
\hline $\begin{array}{l}\text { Immune } \\
\text { Cell }\end{array}$ & Effect & Impact Factor & Source of EVs & Reference \\
\hline \multirow[t]{12}{*}{$\mathrm{T}$ cell } & \multirow[t]{2}{*}{ Inhibit T-cell activation } & CD39, CD73 & Various cancers exosomeal & {$[17]$} \\
\hline & & PD-LI & Glioblastoma EVs & {$[18]$} \\
\hline & Inhibit T-cell proliferation & TGF- $\beta$ & Breast cancer cell exosomal & {$[16]$} \\
\hline & Promote T-cell apoptosis & Fas $\mathrm{L}$ & Ovarian cancer exosomal & [23] \\
\hline & \multirow[t]{3}{*}{$\begin{array}{l}\text { Dysfunction; inhibit ThI and ThI7 } \\
\text { differentiation; suppress T-cell } \\
\text { functions }\end{array}$} & $\begin{array}{l}\text { miR-24-3p, miR-89la, miR- } \\
\text { I06a-5, miR-20a-5p, miR- } \\
\text { I908 }\end{array}$ & Nasopharyngeal carcinoma exosomal & {$[26]$} \\
\hline & & PD-LI & Melanoma/lung/breast cancer exosomal & {$[30]$} \\
\hline & & miR-23a-3p & Hepatocellular carcinoma cells exosomal & [29] \\
\hline & \multirow{4}{*}{$\begin{array}{l}\text { Activate Tregs; promote Treg } \\
\text { expansion }\end{array}$} & CCL20 & Nasopharyngeal carcinoma exosomal & [33] \\
\hline & & TGF- $\beta$ & Colorectal cancer cell EVs & {$[32]$} \\
\hline & & $\operatorname{miR}-2 \mid 4$ & $\begin{array}{l}\text { Lewis lung cancer and human embryonic } \\
\text { kidney cell line microvesicles }\end{array}$ & [34] \\
\hline & & miR-24-3p & $\begin{array}{l}\text { Nasopharyngeal carcinoma (NPC) cell } \\
\text { lines and patient sera exosomal }\end{array}$ & {$[25]$} \\
\hline & $\begin{array}{l}\text { Enhance the inhibitory effect of } \\
\text { MDSCs on } \gamma \delta T \text { cells }\end{array}$ & miR-2I & $\begin{array}{l}\text { Oral squamous cell carcinoma (OSCC) } \\
\text { cell lines Cal- } 27 \text { and SCC- } 9 \text { exosomal }\end{array}$ & [36] \\
\hline \multirow[t]{3}{*}{ B cell } & \multirow[t]{2}{*}{ Suppression function } & Tumor antigen & Pancreatic cancer exosomal & [38] \\
\hline & & - & $\begin{array}{l}\text { Mycoplasma infects tumor cells } \\
\text { exosomal }\end{array}$ & [40] \\
\hline & Promote Breg expansion & - & Esophageal cancer microvesicles & [39] \\
\hline \multirow[t]{6}{*}{ Macrophages } & \multirow[t]{3}{*}{$\begin{array}{l}\text { Formation of inflammatory } \\
\text { environment before metastasis }\end{array}$} & miR-2I; miR-29a & $\begin{array}{l}\text { Lung cancer cell lines A549 and SK-MES } \\
\text { exosomal }\end{array}$ & {$[43]$} \\
\hline & & Annexin A2, IL-6, TNF- $\alpha$ & Breast cancer exosomal & [44] \\
\hline & & $\begin{array}{l}\text { GCSF, IL-6, IL-8, IL-I } \beta, \text { CCL2, } \\
\text { TNF- } \alpha\end{array}$ & Breast cancer exosomal & {$[63]$} \\
\hline & \multirow[t]{3}{*}{$\begin{array}{l}\text { Induce the polarization of macrophage } \\
\text { to } M 2 \text { phenotype }\end{array}$} & miR-222-3p & $\begin{array}{l}\text { Epithelial ovarian cancer Skov-3 cells } \\
\text { exosomal }\end{array}$ & {$[48]$} \\
\hline & & - & Glioblastoma stem cell exosomal & [50] \\
\hline & & - & Lung cancer cell exosomal & [49] \\
\hline \multirow[t]{4}{*}{ DCs } & \multirow[t]{2}{*}{ Inhibit antigen presentation function } & miR-203 & Pancreatic cancer cell panc-I exosomal & {$[53]$} \\
\hline & & miR-2I2-3p & $\begin{array}{l}\text { Pancreatic cancer cell line PANC-I, } \\
\text { SWI990, BxPC-3 exosomal }\end{array}$ & {$[52]$} \\
\hline & \multirow[t]{2}{*}{ Change the direction of differentiation } & - & Breast cancer/lung cancer exosomal & {$[54]$} \\
\hline & & - & Melanoma/colorectal cancer exosomal & [55] \\
\hline
\end{tabular}

(Continued) 
Table I (Continued).

\begin{tabular}{|c|c|c|c|c|}
\hline $\begin{array}{l}\text { Immune } \\
\text { Cell }\end{array}$ & Effect & Impact Factor & Source of EVs & Reference \\
\hline \multirow[t]{5}{*}{ NK cell } & \multirow{2}{*}{$\begin{array}{l}\text { Down-regulate the expression of the } \\
\text { activating receptor NKG2D }\end{array}$} & MICA*008 & Human cervical cancer exosomal & [58] \\
\hline & & NKG2Dligand & Leukemia/lymphoma exosomal & {$[64]$} \\
\hline & \multirow{3}{*}{$\begin{array}{l}\text { Inhibit NK cell function; impair NK } \\
\text { cell-mediated cytotoxicity }\end{array}$} & TGF- $\beta$ & Acute myeloid leukemia exosomal & {$[23]$} \\
\hline & & $\operatorname{miR}-92 b$ & $\begin{array}{l}\text { Serum exosomal from rat model of } \\
\text { orthotopic liver tumor }\end{array}$ & {$[61]$} \\
\hline & & miR-23a;TGF- $\beta$ & $\begin{array}{l}\text { Multiple tumor cells derived } \\
\text { microvesicles }\end{array}$ & [59] \\
\hline
\end{tabular}

factors IL-10, IL-6, and IL-1 $\beta$ and a reduction in the release of IL-17, IL-2 and IFN- $\gamma$. Therefore, nasopharyngeal carcinoma exosomes can hinder $\mathrm{T}$ cell proliferation, promote $\mathrm{CD}^{+} \mathrm{T}$ cell differentiation into helper $\mathrm{T}$ cells (Th1) and Th17 cells and promote the proliferation of Tregs. $^{25,33}$ In the article reviewed by Yin et al, a high level of miR-214 was found in microvesicles derived from several tumors. By downregulating the expression of PTEN in T cells, miR-214 promotes Treg expansion in the TME, and then Tregs can induce effector $\mathrm{T}$ cell apoptosis and boost tumor growth by releasing high levels of IL- $10 .^{34}$

\section{TEXs Inhibit T Cell Function by Activating MDSCs}

Myeloid-derived suppressor cells (MDSCs) are a group of immature bone marrow cells that can significantly inhibit the $\mathrm{T}$ cell immune response. ${ }^{35} \gamma \delta \mathrm{T}$ cells are a unique lymphocyte population that plays a complicated role and can have a positive or negative role in tumors. Li et al found that in the TME, the partial pressure of oxygen can affect the content of tumor-derived exosomes that further inhibit the function of $\gamma \delta \mathrm{T}$ cells and promote tumor progression. ${ }^{36}$ Compared with exosomes in a normoxic environment, exosomes derived from oral squamous cell carcinoma in a hypoxic environment can enhance the inhibitory effect of MDSCs on $\gamma \delta \mathrm{T}$ cells and promote tumor growth by affecting the miR-21/PTEN/PD-L1 signaling axis.

\section{B Lymphocyte}

B cells are a type of lymphocyte that produce antibodies and mediate the humoral immune response, which also act as antigen-presenting cells to initiate adaptive immune responses. Regulatory B cells (Bregs) are a special subset of $B$ cells that play a negative role in antitumor immune responses. $^{37}$ TEVs can directly inhibit the immune function of B cells or indirectly activate Bregs to impair B cell functions. Exosomes derived from pancreatic cancer cells and the plasma of pancreatic cancer patients carry a large number of tumor antigens, which can trick B cells to generate autoantibody reactions, thereby limiting complement-mediated cytotoxicity. ${ }^{38}$ Microvesicles derived from esophageal cancer can induce naive $\mathrm{B}$ cells to differentiate into Bregs, and Bregs inhibit the proliferation of $\mathrm{CD} 8^{+}$ T cells by secreting TGF- $\beta .{ }^{39}$ Yang's study showed that by infecting tumor cells, mycoplasma employs tumor-derived exosomes to deliver their own components and regulate immune cell activity. These mycoplasma components carried by exosomes can specifically activate $\mathrm{B}$ cells in the spleen, induce spleen cells to produce IFN- $\gamma$ and IL-10 factors, and suppress the activity of effector $\mathrm{T}$ cells by activating Breg cells $^{40}$ (Figure 1).

\section{Macrophages}

As the primary effector cells in innate immunity, macrophages not only eliminate antigenic and foreign bodies through strong phagocytosis but also participate in adaptive immune responses as full-time antigen-presenting cells. In different tissue environments, macrophages can differentiate into two diverse polarization states, the classically activated M1 type and the selectively activated M2 type. ${ }^{41}$ M1 macrophages are induced by stimulation with bacterial lipopolysaccharide (LPS) and IFN- $\gamma$ and can then eliminate the pathogenic infection by secreting proinflammatory cytokines and chemokines. While M2 macrophages have anti-inflammatory effects, they can promote angiogenesis and accelerate tumor progression. ${ }^{42}$ 


\section{TEVs Damage Innate Immunity by Regulating Macrophage Function}

In addition to binding with and regulating the expression of targeted mRNA in recipient cells, miRNAs can also act as ligands and play a regulatory role. One study showed that miR-21 and miR-29a carried by lung cancer exosomes can trigger nuclear transcription factor nuclear factor- $\mathrm{KB}$ (NF-kB)-mediated inflammation by acting on human macrophage Toll-like receptor 8 (TLR8) or mouse macrophage TLR7, respectively, and further enhance the secretion of IL-6 and tumor necrosis factor $\alpha$ (TNF- $\alpha$ ) in macrophages, which plays a positive role in the growth and metastasis of lung cancer. ${ }^{43}$ Proteomics analysis revealed that Annexin II is one of the highly expressed proteins in exosomes. Annexin II loaded on breast cancer exosomes increases the secretion of TNF- $\alpha$ and IL- 6 from macrophages by activating the p38/MAPK, NF- $\mathrm{kB}$ and STAT3 signaling pathways, which provides favorable conditions for angiogenesis and tumor metastasis. ${ }^{44} \mathrm{It}$ is worth mentioning that TLR2 and MyD88 are essential "signal adaptors" of the NF- $\mathrm{BB}$ pathway, and ablating TLR2 or MyD88 at the gene level can completely eliminate the tumor-promoting effects of breast cancer-derived exosomes. ${ }^{45}$ Another study demonstrated that in the human body, lung cancer exosomes can participate in the regulation of endogenous cell signaling pathways and reduce the production of IFN- $\gamma$ by delivering activated epidermal growth factor receptor (EGFR) to macrophages, damaging inherent immunization and causing immunosuppression. ${ }^{46}$

\section{TEVs Drives Macrophages Polarizing into M2}

Based on the ability to transport "cargo", exosomes have the potential and ability to regulate the phenotype and polarization of macrophages. ${ }^{47}$ By downregulating the expression of suppressor of cytokine signaling 3 (SOCS3) in macrophages, miR-222-3p packaged in epithelial ovarian exosomes can trigger macrophage polarization toward the M2 phenotype. By secreting proangiogenic factors, M2 macrophages can stimulate tumor growth by promoting angiogenesis and lymphangiogenesis. ${ }^{48}$ Similarly, lung cancer cellderived exosomes can promote M0 macrophage differentiation into the M2 phenotype by enhancing their oxygen consumption rate and altering their bioenergetic state. ${ }^{49}$ Through the STAT3 signaling pathway, glioblastoma stem cell-derived exosomes can mediate the polarization of monocytes into the immunosuppressive M2 type and enhance the expression of PD-L1 on monocytes, thereby impairing antitumor immunity in the $\mathrm{TME}^{50}$ (Figure 1).

\section{Dendritic Cells}

Dendritic cells (DCs) are critical antigen-presenting cells with many dendritic protrusions at maturity; they can recognize, ingest, process foreign antigens, and induce $\mathrm{T}$ cell activation and proliferation by providing antigen peptides. ${ }^{51}$ Furthermore, DCs can regulate the functions of other immune cells by secreting a variety of cytokines and chemokines. Studies have shown that TEVs can promote tumor growth and metastasis by inhibiting the antigen presentation function of DCs in the TME. For instance, exosomal miR-212-3p ${ }^{52}$ and miR-203 ${ }^{53}$ in pancreatic cancer can downregulate the expression of major histocompatibility complex (MHC)-II transcription factor regulatory factor $\mathrm{X}$ associated protein (RFXAP), Toll-like receptor 4 (TLR4) and downstream cytokines in DCs to induce their immune tolerance capacity and promote pancreatic cancer escape from antitumor immunity. By mediating PD-L1 expression, exosomes derived from lung cancer and breast cancer cells can act on myeloid precursor cells to inhibit their differentiation into CD11 $\mathrm{c}^{+}$DCs and promote the formation of an immunosuppressive microenvironment. ${ }^{54}$ Meanwhile, melanoma and colorectal exosomes can induce $\mathrm{CD} 14^{+}$monocytes to differentiate into MDSCs rather than DCs, ${ }^{55}$ thus reducing the antitumor immune response (Figure 1).

\section{Natural Killer Cells}

Natural killer (NK) cells are large granular lymphocytes with the phenotype of $\mathrm{CD}^{-} 6^{+} \mathrm{CD} 3^{-}$that can directly kill target cells without MHC restriction. Under pathological conditions, NK cells can kill cancerous and infected cells independent of antigen presentation and antibody mediation. Hence, NK cells are the first line of defense against infection and tumors in the human body. ${ }^{56}$

\section{TEVs Carrying NKG2D Ligand Inhibit NK Cell Activity}

NK group 2 member D (NKG2D) is an important activating receptor expressed on the NK cell surface. Through carrying NKG2D ligand and releasing TGF- $\beta$, acute myeloid leukemia-derived microvesicles can directly decrease NKG2D expression and suppress the activity and cytotoxicity of NK cells. ${ }^{57}$ The transmembrane glycoproteins MICA/B (MHC class I chain-related proteins $\mathrm{A} / \mathrm{B}$ ) are 
the ligands for NKG2D; unlike other MHC class I proteins, they cannot bind antigen peptides but bind to the NKG2D of NK cells. Soluble MICA/B or exosomal MICA/B can suppress NK cytotoxicity by inhibiting the expression of NKG2D and promoting tumor immune escape. $^{58}$

TEVs Carrying Immunosuppressive Factors Inhibit NK Cytotoxicity

Several studies have shown that TEVs can inhibit NK cell function by delivering TGF- $\beta$ and miRNA. ${ }^{59-61}$ For example, the atypical serine/threonine kinase mammalian target of rapamycin (mTOR) is an important regulator of cell growth and metabolism, which promotes anabolic processes such as ribosome biogenesis and the synthesis of proteins, nucleotides, fatty acids and lipids and inhibits catabolic processes such as autophagy. ${ }^{62}$ By delivering TGF- $\beta$ to inhibit the activation of the serine and threonine kinase mTOR, several tumor-derived exosomes can impair NK cell activity and cytotoxicity. ${ }^{60}$ Similarly, miR-92b ${ }^{61}$ derived from hepatic carcinoma exosomes can inhibit the expression of CD69 on NK cells and inhibit NK cellmediated cytotoxicity. Compared with those secreted in a normal oxygen environment, tumor microvesicles secreted in a hypoxic environment have a more significant inhibitory effect on NK cells. Multiple tumor-derived microvesicles can deliver a large quantity of immunosuppressive factors, such as miR-23a and TGF- $\beta$, to NK cells to suppress the expression of CD107a and NKG2D on NK cells and further inhibit NK cell activation and function ${ }^{59}$ (Figure 1).

\section{The Application of TEVs in Tumor Immunotherapy}

Currently, nanotechnology has been widely used in cancer treatment. Several synthetic nanoparticles, such as pegaspargase $^{65}$ and doxorubicin, ${ }^{66}$ have been approved by the FDA for treating acute lymphoblastic leukemia, advanced ovarian cancer, and multiple bone marrow cancers clinically. Although synthetic nanoparticles have the advantages of easy manufacture, separation and purification with high yields, they still have many limitations, including high toxicity, lack of targeting specificity, rapid elimination by the mononuclear phagocytic system, and low bioavailability. ${ }^{67}$ Compared with synthetic nanoparticles, EVs are natural vehicles for cargo delivery. Because of their unique biocompatibility, minimal toxicity, high stability and cell targeting ability, ${ }^{68}$ EVs have great potential for targeted drug delivery.
In addition, TEVs can also be developed and applied as tumor diagnostic markers and vaccines because they carry tumor-associated antigen (TAA) and so on (Figure 2).

\section{TEVs: Potential Biomarkers for Tumor Diagnosis}

EVs can be extracted from an extremely wide range of sources. In addition to in cell culture supernatant, EVs also largely exist in biological fluids such as blood, urine, saliva, and sweat, ${ }^{7}$ which makes them easy to obtain and analyze for liquid biopsies. Currently, many clinical trials are focusing on evaluating the components of TEVs, including DNA, miRNA, long noncoding RNA (lncRNA), and certain tumor antigens, as biomarkers used in cancer diagnostics, which are detailed in Table 2. Meanwhile, TEVs can also reflect the effect of drug treatment at different tumor stages. An ongoing clinical trial is evaluating the expression profile of exosomal miRNAs and PD-L1 in non-small cell lung cancer (NSCLC) patients before and after immunotherapy and exploring the potential of exosomes as biomarkers to predict the curative effect of anticancer drugs (pabolizumab/ nafulizumab) (NCT04427475).

However, how to efficiently separate EVs is still an urgent issue that needs to be solved. Furthermore, a method for the accurate quantification of EVs is also necessary for the clinical application of EVs in the future. At present, the separation methods of EVs mainly include the kit method, the immunomagnetic bead method, ultracentrifugation and ultrafiltration centrifugation, but these methods all still have many drawbacks. ${ }^{45}$ They are either expensive or extremely dependent on specific instruments and take a long time. Recently, a study ${ }^{69}$ demonstrated the development of a new simple method for directly isolating and quantifying disease-specific exosomes by using carboxyl group-functionalized iron oxide nanoparticles (C-IONPs). This method includes two steps. First, functional C-IONPs that carry exosome marker proteins (tetraspanin, CD9, etc.) are used to separate bulk exosomes from the initial sample; then, the pathogen-specific exosomes are preliminarily separated from the sample by electrochemical methods and quantified by tumor-specific markers. Electrochemical methods have excellent sensitivity and specificity for the detection of biomolecules in biological substrates and have been applied to quantify exosomes derived from ovarian cancer and breast cancer. $^{70}$ 


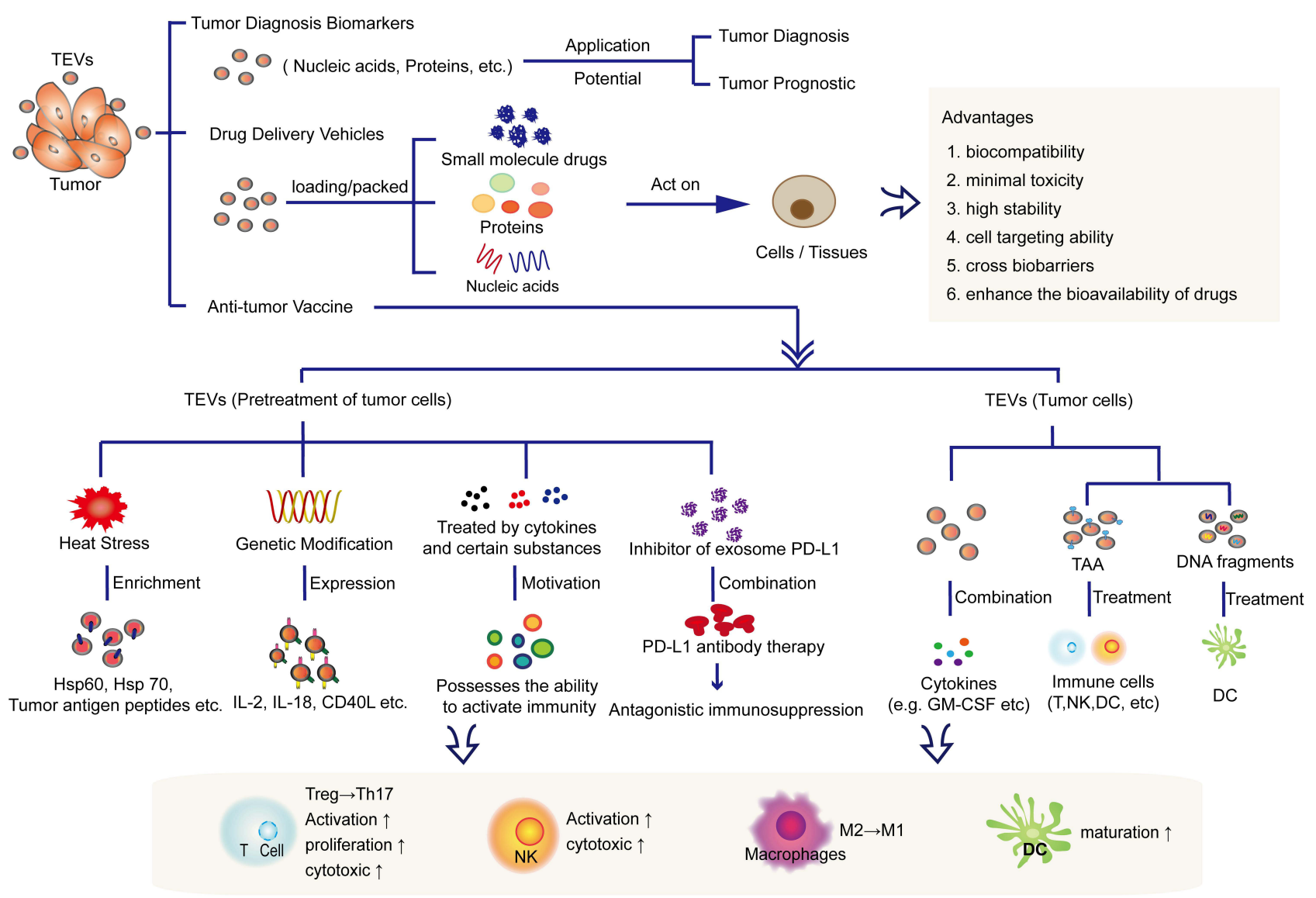

Figure 2 Application of TEVs in tumor diagnosis, drug vesicles and immunotherapy. TEVs can not only be used as a biomarkers or drug delivery vehicle due to its own advantages, but also can be used as a tumor vaccine for tumor immunotherapy.

\section{TEVs: Drug Delivery Vehicles}

EVs are natural delivery vehicles that not only have the ability to cross biobarriers (cytoplasmic membrane and blood-brain barrier) but also have inherent long-term circulation capabilities and excellent biocompatibility. Compared to other drug carriers, such as lipidosomes and viruses, EVs induce a lesser immune response, and their drug transfer efficiency is also excellent. ${ }^{71}$ They have been used to deliver nucleic acids, proteins and small molecule drugs to targeted cells or tissues in oncotherapy. ${ }^{15,72,73}$ For example, one study showed that EVs derived from patient body fluids can be modified by electroporation to carry exogenous siRNA and then transport the siRNA to human blood cells (monocytes and lymphocytes). ${ }^{72}$ A variety of small molecule anticancer drugs and natural compounds (such as paclitaxel, curcumin, doxorubicin, celastrol, and $\beta$-elemene) have been packed in exosomes to enhance the bioavailability of drugs and inhibit tumor growth and metastasis. ${ }^{74}$ To date, related studies have mainly focused on using vesicles derived from stem cells, but some researchers have attempted to use TEVs as carriers to deliver antitumor drugs. Table 3 summarizes recent studies that focused on TEVs as carriers for tumor treatment. For example, loading paclitaxel to autologous prostate cancer cell ( $\mathrm{LNCaP}$ and $\mathrm{PC}-3$ )-derived exosomes increased the cytotoxic effect of paclitaxel on prostate cancer. ${ }^{75}$ Gang Jia's team ${ }^{76}$ designed a new type of TEV for glioma treatment, which was not only loaded with superparamagnetic iron oxide nanoparticles (SPIONs) and the chemical drug curcumin but also combined with exosomal membranes and the gliomatargeting ligand RGERPPR peptide (RGE) by click chemistry methods. These types of TEVs showed ideal antiglioma effects and provide a potential method for the diagnosis and treatment of intracranial tumors. In general, by combining the biological properties of exosomes and preparing synthetic nanoparticles, the clinical application of nanomaterial-based drug delivery systems will be greatly promoted and developed. 
Table 2 Clinical Trials on Using TEVs for Tumor Diagnosis Biomakers

\begin{tabular}{|c|c|c|c|c|}
\hline Cancer Type & Vesicles & Sample & ID & Apply \\
\hline Colorectal cancer & TEXs proteins & Blood & NCT04394572 & Early diagnosis \\
\hline Colorectalcancer & TEXs miRNA & Blood & NCT04523389 & Early prognostic \\
\hline Lung cancer & Exosomes & Blood plasma & NCT045299I5 & Early diagnosis \\
\hline HER2-positive breast cancer & Exosomes & Blood and tumor tissue & NCT04288I4I & Diagnosis \\
\hline Prostate cancer & $\begin{array}{l}\text { Exosomes } \\
\text { gene }\end{array}$ & Urinary & NCT02702856 & Diagnosis \\
\hline Clear cell renal cell carcinoma & Exosomes & Urinary & NCT04053855 & Early diagnosis \\
\hline Pancreatic cancer & Exosomes & Blood and tissue & NCT02393703 & Diagnosis \\
\hline Bladder cancer & Exosomes & Urinary & NCT04I55359 & Diagnosis \\
\hline Lung cancer & Exosomes & Blood & NCT04629079 & Early diagnosis \\
\hline Prostate cancer & Exosomes & Blood & NCT04556916 & Early diagnosis \\
\hline $\begin{array}{l}\text { Papillomavirus-positive oropharyngeal squamous cell } \\
\text { carcinoma }\end{array}$ & Exosomes & Blood and saliva & NCT02।474I8 & Screening \\
\hline Rectal cancer & Exosomes & Blood & NCT03874559 & Diagnosis \\
\hline Lung cancer & TEXs IncRNA & Serum & NCT038306I9 & Early diagnosis \\
\hline Metastatic breast cancer & Exosomes & Blood/tissues & NCT04258735 & $\begin{array}{l}\text { Genomic } \\
\text { analysis }\end{array}$ \\
\hline Digestive and gynecologic/breast cancer & TEXs DNA & Blood & NCT04530890 & Diagnosis \\
\hline Prostate cancer & Exosomes & Blood & NCT03694483 & Diagnosis \\
\hline Rectal cancer & TEVs & Blood & NCT04852653 & Liquid biopsy \\
\hline NSCLC & Exosomes & Blood & NCT04499794 & Prognostic \\
\hline
\end{tabular}

\section{TEVs: Antitumor Vaccines}

TEVs have the potential to become cancer vaccines. The previous article has reviewed in detail the inhibitory effect of TEVs on immune cells. Then, how to block this inhibitory effect to restore the activity of immune cells and use the "tumor-specific components" of TEVs to activate immune cells are the key to the development of tumor vaccines. From the past to the present, many researchers have devoted themselves to exploring the anti-tumor effects of tumor-derived extracellular vesicles and the possibility of applying them to future clinical treatments. At present, numerous studies in vivo and in vitro have confirmed the possibility of tumor-derived extracellular vesicles as tumor vaccines. The details are described below. However, if it is applied to the clinical treatment of tumors in the future, there are still many problems and difficulties that need to be solved urgently. Of course, until now, the distinction and separation of extracellular vesicle subsets is still the primary problem to be solved in this field. Only after the extracellular vesicles are accurately separated, the characteristics and markers of each subset can be deeply understood. Then, by comparing the similarities and differences between them, the role of each subset in cellular communication and physiological processes can be clarified.

\section{Heat Stress and Heat Shock Protein}

Compared with exosomes secreted under a normal environment, tumor-derived exosomes secreted under heatstressed circumstances can induce specific antitumor immune responses more effectively. ${ }^{79}$ Under heat stress circumstances, tumor cells can produce and release exosomes enriched in heat shock proteins such as Hsp60 and 
Table 3 Study of TEVs as Drug Vehicles

\begin{tabular}{|c|c|c|c|c|c|c|}
\hline Drugs & Source & Type & $\begin{array}{l}\text { Delivery } \\
\text { Methods }\end{array}$ & Targets & Effect & Reference \\
\hline Paclitaxel & $\begin{array}{c}\text { Prostate cancer LNCaP and } \\
\text { PC-3 cells }\end{array}$ & Exosomes & Incubation & $\begin{array}{l}\text { Autologous prostate } \\
\text { cancer cells }\end{array}$ & $\begin{array}{l}\text { Increased the anti-tumor } \\
\text { effect of Paclitaxel }\end{array}$ & {$[75]$} \\
\hline Curcumin & $\begin{array}{c}\text { Pancreatic adenocarcinoma } \\
\text { cell (PANC-I, MIA PaCa-2) } \\
\text { derived exosomes }\end{array}$ & Exosomes & $\begin{array}{l}\text { Tumors cells } \\
\text { which } \\
\text { treatment with } \\
\text { drugs }\end{array}$ & PANC-I; MIA PaCa-2 & $\begin{array}{c}\text { Promoting anti-tumor } \\
\text { effect reduces tumor cells } \\
\text { viability }\end{array}$ & [77] \\
\hline siRNA & Plasma exosomes & Exosomes & Electroporation & $\begin{array}{l}\text { Monocytes; } \\
\text { lymphocytes }\end{array}$ & $\begin{array}{l}\text { Provide cells with } \\
\text { heterologous nucleic acids } \\
\text { such as therapeutic siRNAs }\end{array}$ & [72] \\
\hline Paclitaxel & Glioblastoma cell line U-87 & Exosomes & $\begin{array}{l}\text { Incubation; } \\
\text { sonication }\end{array}$ & U-87 & $\begin{array}{l}\text { Increased drugs effect } \\
\text { against U-87 cell line }\end{array}$ & [78] \\
\hline Curcumin & $\begin{array}{l}\text { Mouse macrophage cell line } \\
\text { Raw } 264.7\end{array}$ & Exosomes & Electroporation & $\begin{array}{l}\text { Human glioma cell line } \\
\text { U25I; human hepatoma } \\
\text { cell line Bel-7404 }\end{array}$ & $\begin{array}{l}\text { Provided good results for } \\
\text { targeted imaging and } \\
\text { therapy of glioma. }\end{array}$ & {$[76]$} \\
\hline
\end{tabular}

Hsp70 as well as tumor antigen peptides ${ }^{80}$ to activate the antitumor effect of immune cells. In addition, heat stressinduced tumor exosomes are rich in chemokines CCL20, CCL5, CCL4, CCL3 and CCL2, which can activate

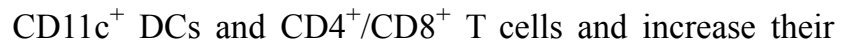
chemotaxis capacity. ${ }^{79}$ It has been reported that in a heatstressed environment, colorectal-derived exosomes ${ }^{81}$ can induce Treg cells to transform into Th17 cells in the presence of IL-6, thereby producing strong antitumor effects in vitro. Through engineering methods, Xie et al expressed Hsp 70 on the cell membrane of myeloma cells to simulate heat stress. The exosomes obtained from the myeloma cells carried a large amount of Hsp70, which could activate $\mathrm{CD}^{+} \mathrm{T}$ and NK cells in vitro. ${ }^{80}$

\section{Tumor-Associated Antigen and DNA}

TEVs have been demonstrated to be able to carry TAA, ${ }^{82}$ which can be used as a pivotal component of antitumor vaccines in cancer therapy. One study ${ }^{83}$ revealed that exosomes derived from renal cell carcinoma carrying a large amount of TAAs, through Fas/FasL signaling pathway mediation, can provoke forceful cytotoxic effects from $\mathrm{CD}^{+} \mathrm{T}$ cells against autologous tumor cells in vivo. Similarly, exosomes extracted from the serum of HCC patients can be used as specific HCC antigens. These HCC patient serum-derived exosomes were proven to significantly inhibit tumors in orthotopic HCC mice pulsed with DC treatment. HCC exosomes can transfer their antigens to DCs, and these preimmunized DCs can elicit antitumor immunity by significantly promoting the proliferation of specific $\mathrm{CD}^{+} \mathrm{T}$ cells in mice. Furthermore, HCC exosome treatment with DCs increased INF- $\gamma$ levels, decreased IL-10 and TNF- $\beta$ levels in the TME and significantly inhibited tumor growth in a mouse model.

The DNA fragments derived from parental cells account for a substantial component of exosomal cargoes. Through an unknown mechanism, these DNA fragments that originate from the nuclei and mitochondria accumulate in the cytoplasm and are then packaged into exosomes. ${ }^{84}$ The CD47 protein is an immune checkpoint receptor that can interact with ligand signal regulatory protein alpha (SIRP $\alpha$ ) on innate immune cells to transmit negative signals to inhibit macrophage-mediated phagocytosis. $^{85}$ One study revealed that blocking CD47 expression in tumor cells can cause preferential accumulation and export of tumor mitochondrial DNA by exosomes, which activates STING signaling in neighboring DCs to promote antitumor immunity. ${ }^{86}$

\section{Treatment and Modification of Tumor Cells}

A series of studies have revealed that by pretreating tumor cells with cytokines and certain substances with antitumor effects, TEVs can be used to develop antitumor vaccines. For example, the epigallocatechin gallate (EGCG) present in green tea has been shown to have an inhibitory effect on tumorigenesis in vivo and in vitro. ${ }^{87}$ After EGCG 
treatment, exosomes derived from 4T1 cells were capable of altering the phenotype of tumor-associated macrophages (TAMs) from the M2 to the M1 phenotype. ${ }^{88}$ Recently, Yang et al found ${ }^{89}$ that interferon regulatory factor 1 (IRF-1) can upregulate the expression of IL$15 \mathrm{R} \alpha$ and MHC-I on Hepa1-6 and MC38 cell (two mouse tumor cell lines)-derived exosomes. Exosomes with high IL-15R $\alpha$ and MHC-I expression can improve the antitumor effect in mice by promoting $\mathrm{CD}^{+}$and $\mathrm{CD} 8 \alpha^{+} \mathrm{T}$ cell infiltration into tumors. Similarly, combined with granulocyte-macrophage colony stimulating factor (GM-CSF), ascites-derived exosomes ${ }^{90}$ from colorectal cancer patients can induce specific antitumor responses in cytotoxic $\mathrm{T}$ lymphocytes. In addition, by overexpressing the genes encoding IL-2 or IL-18 in tumor cells, TEVs carrying IL-2 or IL-18 were generated by the Yunshan ${ }^{91}$ and Shengming ${ }^{92}$ research groups, respectively. Their results indicated that TEVs carrying IL-2 can induce the polarization of antigen-specific TH1 cells and activate the cytotoxicity of $\mathrm{T}$ cells, while TEVs carrying IL-18 can promote DC maturation and the proliferation of cytotoxic T cells. CD40 signaling plays a vital role in inducing DC maturation and the antitumor immune response. In a study93 by Wang et al, through transfection of the CD40 L gene into lung cancer cells, CD40L-carrying exosomes were obtained, which can effectively promote the maturation of DCs and the activity of antitumor $\mathrm{T}$ cells in vitro. These data indicated that it is worth further studying and developing an effective antitumor vaccine strategy based on exosomes obtained from overexpressing specific proteins in tumor cells.

\section{Strategy to Impair the Immunosuppressive Effects of TEVs}

Because of the immunosuppressive property of TEVs, it can be assumed that impairing the immunosuppressive ability of TEVs may produce better therapeutic effects when they are used as drug delivery vehicles. One study $^{94}$ revealed that by using small molecule inhibitors against NSMNASE2 and RAB27A, the expression of exosomal PD-L1 can be blocked in prostate cancer cell lines and melanoma cell lines. When performing small molecule inhibitor treatment accompanied by PD-L1 antibody therapy simultaneously, cancer patients who were not sensitive to PD-L1 antibody treatments achieved better therapeutic effects. An interesting assumption is that an in situ PD-L1 blockade therapy strategy can synergistically induce systemic immune responses in multiple tumor sites.
Therefore, blocking the expression of exosomal PD-L1 may be a new immunotherapy strategy to improve the effect of antibody therapy in specific tumor types, such as prostate cancer and melanoma.

\section{Discussion}

Now, there are still many biological challenges and issues related to the role of TEVs in immune cells and immunotherapy, tumor diagnosis and drug delivery vehicles. The first difficulty is that subsets of EVs such as microvesicles and exosomes are difficult to separate accurately. The commonly used methods have been reviewed above and will not be repeated here. Nowadays, interdisciplinary is more and more common. The combination of materials science, chemistry and other disciplines with biology also brings new breakthroughs for the precise separation of extracellular vesicles. For example, a research team ${ }^{95}$ from Fudan University and Ningbo University has developed a microfluidic Raman biochip for the separation and analysis of exosomes, which has been successfully applied to the determination of exosomes in clinical serum samples. This biochip has potential to become a promising tool for prostate cancer diagnosis.

Extracellular vesicles are rich in nucleic acids, proteins and other substances from parental cells. Nucleic acids, especially miRNAs, have been extensively studied. Certainly, the study of TEVs proteome is equally important. For example, the TEVs protein group can be compared with the tumor protein group, which is conducive to discovering important tumor-related proteins (such as proteins that can regulate immune cells and self-activity) and tumor markers which can be used as therapeutic targets or applied to tumor diagnosis.

Although studies have shown that TEVs carrying tumorspecific antigens and nucleic acids can act to activate immune cells, the current research on exosomes is still very superficial. For in vitro experiments, in addition to conventional cells proliferation, apoptosis, cycle and other experimental indicators, the secretion level and composition changes of TEVs should also be studied. For example, does the cell growth period and cell state affect the secretion of EV? Is there any change in the composition of EVs? If there is a change, how much can it be? These can further help researchers to deepen their understanding of tumors.

Immunotherapy is a major research hotspot in cancer treatment in recent years. It is mainly divided into somatic immunotherapy and immune checkpoint inhibitor therapy. Among them, immune checkpoint inhibitor 
anti-PD-1 and anti-PD-L1 monoclonal drugs have been extensively studied and have been used in clinical tumor treatment. However, this type of treatment is not suitable for all cancers. It can only treat and relieve a small proportion of patients in certain cancer types. In recent years, studies ${ }^{96}$ have found that TEVs also carry PD-L1 on the surface, which can induce immune escape and promote tumor progression, such as TEVs PD-L1 mediates resistance to immunotherapy by directly binding to anti-PD-L1 antibodies. Therefore, PD-L1 on the surface of TEVs: 1) as a target to develop more effective immune checkpoint inhibitors; 2) as a research object to in-depth study of the occurrence and mechanism of PD-L1.

\section{Conclusion and Perspectives}

With the rapid progress of social modernization and industrialization, the pace of life is accelerating gradually. Excessive pressures and serious environmental pollution are the latent reasons for the increasing tumor incidence. Through several mechanisms, tumors can escape immune surveillance and clearance to boost their occurrence, development and metastasis. Therefore, it is urgent to understand the underlying mechanism of tumor immune escape.

As a mediator between tumors and immune cells, TEVs play a dual role in modulating immune cells in the TME. On the one hand, TEVs can inhibit the activation, proliferation, differentiation and function of immune cells by directly acting on them or promoting the activity of immunosuppressive cells. On the other hand, TEVs can also act as specific tumor antigens to activate immune cells, promote antitumor immunity and inhibit tumor growth. Therefore, how to circumvent the immunosuppressive effect of TEVs and use their immune stimulus activation is the key research direction in the future. Of course, regardless of their effects on immune cells, activation or suppression, TEVs can still be used as specific targets for tumor recognition and treatment. Further identification of specific tumor markers on TEVs will allow for early diagnosis and prognostic analysis for tumor patients in the clinic.

At present, there are still many limitations hindering research on TEVs, such as the lack of precise separation and identification methods and efficient isolation methods for TEVs. Thus, how to rationally use the advantages of TEVs while weakening or avoiding their side effects will be the research focus in the tumor therapy field. In addition, in-depth exploration of the role of TEVs in modulating immune cells is the goal of researchers who focus on tumor immunity, which can provide new inspiration for tumor treatment.

\section{Ethics Statement}

This paper is a review article without any in vitro or in vivo study.

\section{Funding}

This work was funded by the China Postdoctoral Science Foundation (grant number 2020M673491); Fundamental Research Funds for the Central Universities (grant number 3102020smxy007); and the Training Program of Innovation and Entrepreneurship for Undergraduates (grant number S202010699401).

\section{Disclosure}

$\mathrm{J}$ Chen and F Han are the employees of Shanxi Weiqidaguangming Pharmaceutical Co., Ltd. The authors report no other possible conflicts of interest in this work.

\section{References}

1. Théry C, Witwer KW, Aikawa E, et al. Minimal information for studies of extracellular vesicles 2018 (MISEV2018): a position statement of the International Society for Extracellular Vesicles and update of the MISEV2014 guidelines. J Extracell Vesic. 2018;7 (1): 1535750 .

2. Greening DW, Simpson RJ. Understanding extracellular vesicle diversity - current status. Expert Rev Proteomics. 2018;15 (11):887-910.

3. van Niel G, D'Angelo G, Raposo G. Shedding light on the cell biology of extracellular vesicles. Nat Rev Mol Cell Biol. 2018;19 (4):213-228.

4. Zhang H, Freitas D, Kim HS, et al. Identification of distinct nanoparticles and subsets of extracellular vesicles by asymmetric flow field-flow fractionation. Nat Cell Biol. 2018;20(3):332-343.

5. McAndrews KM, Kalluri R. Mechanisms associated with biogenesis of exosomes in cancer. Mol Cancer. 2019;18(1):52.

6. Yáñez-Mó M, Siljander PR, Andreu Z, et al. Biological properties of extracellular vesicles and their physiological functions. $J$ Extracell Vesic. 2015;4:27066.

7. Pegtel DM, Gould SJ. Exosomes. Annu Rev Biochem. 2019;88:487-514.

8. Rahbarghazi R, Jabbari N, Sani NA, et al. Tumor-derived extracellular vesicles: reliable tools for cancer diagnosis and clinical applications. Cell Commun Signal. 2019;17(1):73.

9. Guo S, Deng CX. Effect of stromal cells in tumor microenvironment on metastasis initiation. Int J Biol Sci. 2018;14(14):2083-2093.

10. Costa-Silva B, Aiello NM, Ocean AJ, et al. Pancreatic cancer exosomes initiate pre-metastatic niche formation in the liver. Nat Cell Biol. 2015;17(6):816-826.

11. Li W, Li C, Zhou T, et al. Role of exosomal proteins in cancer diagnosis. Mol Cancer. 2017;16(1):145.

12. Whiteside TL. Tumor-derived exosomes and their role in tumor-induced immune suppression. Vaccines. 2016;4(4):35.

13. Maybruck BT, Pfannenstiel LW, Diaz-Montero M, Gastman BR. Tumor-derived exosomes induce $\mathrm{CD} 8(+) \mathrm{T}$ cell suppressors. J Immunother Cancer. 2017;5(1):65. 
14. Parkin J, Cohen B. An overview of the immune system. Lancet (London, England). 2001;357(9270):1777-1789.

15. Taghikhani A, Farzaneh F, Sharifzad F, Mardpour S, Ebrahimi M, Hassan ZM. Engineered tumor-derived extracellular vesicles: potentials in cancer immunotherapy. Front Immunol. 2020;11:221.

16. Rong L, Li R, Li S, Luo R. Immunosuppression of breast cancer cells mediated by transforming growth factor-beta in exosomes from cancer cells. Oncol Lett. 2016;11(1):500-504.

17. Clayton A, Al-Taei S, Webber J, Mason MD, Tabi Z. Cancer exosomes express CD39 and CD73, which suppress $\mathrm{T}$ cells through adenosine production. $J$ Immunol. 2011;187(2):676-683.

18. Ricklefs FL, Alayo Q, Krenzlin H, et al. Immune evasion mediated by PD-L1 on glioblastoma-derived extracellular vesicles. Sci $A d v$ 2018;4(3):eaar2766.

19. Theodoraki MN, Yerneni SS, Hoffmann TK, Gooding WE, Whiteside TL. Clinical significance of PD-L1(+) exosomes in plasma of head and neck cancer patients. Clin Cancer Res. 2018;24 (4):896-905.

20. Okuyama K, Michi Y, Mizutani M, Yamashiro M, Kaida A, Harada K. Clinical study on mandibular fracture after marginal resection of the mandible. Oral Surg Oral Med Oral Pathol Oral Radiol. 2016;121(5):461-467.

21. Dermani FK, Samadi P, Rahmani G, Kohlan AK, Najafi R. PD-1/PDL1 immune checkpoint: potential target for cancer therapy. $J$ Cell Physiol. 2019;234(2):1313-1325.

22. Filipazzi P, Burdek M, Villa A, Rivoltini L, Huber V. Recent advances on the role of tumor exosomes in immunosuppression and disease progression. Semin Cancer Biol. 2012;22(4):342-349.

23. Whiteside TL. Immune modulation of T-cell and NK (natural killer) cell activities by TEXs (tumour-derived exosomes). Biochem Soc Trans. 2013;41(1):245-251.

24. Leichter AL, Sullivan MJ, Eccles MR, Chatterjee A. MicroRNA expression patterns and signalling pathways in the development and progression of childhood solid tumours. Mol Cancer. 2017;16(1):15.

25. Ye SB, Zhang H, Cai TT, et al. Exosomal miR-24-3p impedes T-cell function by targeting FGF11 and serves as a potential prognostic biomarker for nasopharyngeal carcinoma. J Pathol. 2016;240(3):329-340.

26. Ye SB, Li ZL, Luo DH, et al. Tumor-derived exosomes promote tumor progression and T-cell dysfunction through the regulation of enriched exosomal microRNAs in human nasopharyngeal carcinoma. Oncotarget. 2014;5(14):5439-5452.

27. Wang X, Shen H, Zhangyuan G, et al. 14-3-3zeta delivered by hepatocellular carcinoma-derived exosomes impaired anti-tumor function of tumor-infiltrating $\mathrm{T}$ lymphocytes. Cell Death Dis. 2018;9(2):159.

28. Yang Y, Li CW, Chan LC, et al. Exosomal PD-L1 harbors active defense function to suppress $\mathrm{T}$ cell killing of breast cancer cells and promote tumor growth. Cell Res. 2018;28(8):862-864.

29. Liu J, Fan L, Yu H, et al. Endoplasmic reticulum stress causes liver cancer cells to release exosomal miR-23a-3p and up-regulate programmed death ligand 1 expression in macrophages. Hepatology (Baltimore, Md). 2019;70(1):241-258.

30. Chen G, Huang AC, Zhang W, et al. Exosomal PD-L1 contributes to immunosuppression and is associated with anti-PD-1 response. Nature. 2018;560(7718):382-386.

31. Muller L, Simms P, Hong CS, et al. Human tumor-derived exosomes (TEX) regulate Treg functions via cell surface signaling rather than uptake mechanisms. Oncoimmunology. 2017;6(8):e1261243.

32. Yamada N, Kuranaga Y, Kumazaki M, Shinohara H, Taniguchi K, Akao Y. Colorectal cancer cell-derived extracellular vesicles induce phenotypic alteration of $\mathrm{T}$ cells into tumor-growth supporting cells with transforming growth factor- $\beta 1$-mediated suppression. Oncotarget. 2016;7(19):27033-27043.

33. Mrizak D, Martin N, Barjon C, et al. Effect of nasopharyngeal carcinoma-derived exosomes on human regulatory $\mathrm{T}$ cells. $J$ Natl Cancer Inst. 2015;107(1):363.
34. Yin Y, Cai X, Chen X, et al. Tumor-secreted miR-214 induces regulatory T cells: a major link between immune evasion and tumor growth. Cell Res. 2014;24(10):1164-1180.

35. Gabrilovich DI, Nagaraj S. Myeloid-derived suppressor cells as regulators of the immune system. Nat Rev Immunol. 2009;9(3):162-174.

36. Li L, Cao B, Liang X, et al. Microenvironmental oxygen pressure orchestrates an anti- and pro-tumoral $\gamma \delta$ T cell equilibrium via tumorderived exosomes. Oncogene. 2019;38(15):2830-2843.

37. Rosser EC, Mauri C. Regulatory B cells: origin, phenotype, and function. Immunity. 2015;42(4):607-612.

38. Capello M, Vykoukal JV, Katayama H, et al. Exosomes harbor B cell targets in pancreatic adenocarcinoma and exert decoy function against complement-mediated cytotoxicity. Nat Commun. 2019;10(1):254.

39. Li Y, An J, Huang S, He J, Zhang J. Esophageal cancer-derived microvesicles induce regulatory B cells. Cell Biochem Funct. 2015;33(5):308-313.

40. Yang C, Chalasani G, Ng YH, Robbins PD. Exosomes released from Mycoplasma infected tumor cells activate inhibitory B cells. PLoS One. 2012;7(4): 36138

41. Murray PJ. Macrophage polarization. Annu Rev Physiol. 2017;79:541-566.

42. Mantovani A, Sica A. Macrophages, innate immunity and cancer: balance, tolerance, and diversity. Curr Opin Immunol. 2010;22(2):231-237.

43. Fabbri M, Paone A, Calore F, et al. MicroRNAs bind to Toll-like receptors to induce prometastatic inflammatory response. Proc Natl Acad Sci USA. 2012;109(31):E2110-E2116.

44. Maji S, Chaudhary P, Akopova I, et al. Exosomal annexin II promotes angiogenesis and breast cancer metastasis. Mol Cancer Res. 2017;15(1):93-105.

45. Boriachek K, Islam MN, Möller A, et al. Biological functions and current advances in isolation and detection strategies for exosome nanovesicles. Small. 2018;14(6):1702153.

46. Gao L, Wang L, Dai T, et al. Tumor-derived exosomes antagonize innate antiviral immunity. Nat Immunol. 2018;19(3):233-245.

47. Baig MS, Roy A, Rajpoot S, et al. Tumor-derived exosomes in the regulation of macrophage polarization. Inflam Res. 2020;69 (5):435-451.

48. Ying $\mathrm{X}, \mathrm{Wu} \mathrm{Q}, \mathrm{Wu} \mathrm{X}$, et al. Epithelial ovarian cancer-secreted exosomal miR-222-3p induces polarization of tumor-associated macrophages. Oncotarget. 2016;7(28):43076-43087.

49. Pritchard A, Tousif S, Wang Y, et al. Lung tumor cell-derived exosomes promote M2 macrophage polarization. Cells. 2020;9(5):1303.

50. Gabrusiewicz K, Li X, Wei J, et al. Glioblastoma stem cell-derived exosomes induce M2 macrophages and PD-L1 expression on human monocytes. Oncoimmunology. 2018;7(4):e1412909.

51. Palucka K, Banchereau J. Cancer immunotherapy via dendritic cells. Nat Rev Cancer. 2012;12(4):265-277.

52. Ding G, Zhou L, Qian Y, et al. Pancreatic cancer-derived exosomes transfer miRNAs to dendritic cells and inhibit RFXAP expression via miR-212-3p. Oncotarget. 2015;6(30):29877-29888.

53. Zhou M, Chen J, Zhou L, Chen W, Ding G, Cao L. Pancreatic cancer derived exosomes regulate the expression of TLR4 in dendritic cells via miR-203. Cell Immunol. 2014;292(1-2):65-69.

54. Ning Y, Shen K, Wu Q, et al. Tumor exosomes block dendritic cells maturation to decrease the $\mathrm{T}$ cell immune response. Immunol Lett. 2018;199:36-43.

55. Chen W, Jiang J, Xia W, Huang J. Tumor-related exosomes contribute to tumor-promoting microenvironment: an immunological perspective. J Immunol Res. 2017;2017:1073947.

56. Terrén I, Orrantia A, Vitallé J, Zenarruzabeitia O, Borrego F. NK cell metabolism and tumor microenvironment. Front Immunol. 2019;10:2278.

57. Szczepanski MJ, Szajnik M, Welsh A, Whiteside TL, Boyiadzis M. Blastderived microvesicles in sera from patients with acute myeloid leukemia suppress natural killer cell function via membrane-associated transforming growth factor-beta1. Haematologica. 2011;96(9):1302-1309. 
58. Ashiru O, Boutet P, Fernández-Messina L, et al. Natural killer cell cytotoxicity is suppressed by exposure to the human NKG2D ligand MICA*008 that is shed by tumor cells in exosomes. Cancer Res. 2010;70(2):481-489.

59. Berchem G, Noman MZ, Bosseler M, et al. Hypoxic tumor-derived microvesicles negatively regulate $\mathrm{NK}$ cell function by a mechanism involving TGF- $\beta$ and miR23a transfer. Oncoimmunology. 2016;5(4): e1062968.

60. Viel S, Marçais A, Guimaraes FS, et al. TGF- $\beta$ inhibits the activation and functions of NK cells by repressing the mTOR pathway. $S c i$ Signal. 2016;9(415):ra19.

61. Nakano T, Chen IH, Wang CC, et al. Circulating exosomal miR-92b: its role for cancer immunoediting and clinical value for prediction of posttransplant hepatocellular carcinoma recurrence. Am J Transplant. 2019;19(12):3250-3262.

62. Laplante M, Sabatini DM. mTOR signaling in growth control and disease. Cell. 2012;149(2):274-293.

63. Chow A, Zhou W, Liu L, et al. Macrophage immunomodulation by breast cancer-derived exosomes requires Toll-like receptor 2-mediated activation of NF-кB. Sci Rep. 2014;4:5750.

64. Hedlund M, Nagaeva O, Kargl D, Baranov V, Mincheva-Nilsson L. Thermal- and oxidative stress causes enhanced release of NKG2D ligand-bearing immunosuppressive exosomes in leukemia/lymphoma T and B cells. PLoS One. 2011;6(2):e16899.

65. Dinndorf PA, Gootenberg J, Cohen MH, Keegan P, Pazdur R. FDA drug approval summary: pegaspargase (oncaspar) for the first-line treatment of children with acute lymphoblastic leukemia (ALL). Oncologist. 2007;12(8):991-998.

66. Barenholz Y. Doxil ${ }^{\circledR}$-the first FDA-approved nano-drug: lessons learned. J Controll Release. 2012;160(2):117-134.

67. Ullah M, Kodam SP, Mu Q, Akbar A. Microbubbles versus extracellular vesicles as therapeutic cargo for targeting drug delivery. ACS Nano. 2021;15(3):3612-3620.

68. Kibria G, Ramos EK, Wan Y, Gius DR, Liu H. Exosomes as a drug delivery system in cancer therapy: potential and challenges. Mol Pharm. 2018;15(9):3625-3633.

69. Farhana FZ, Umer M, Saeed A, et al. Isolation and detection of exosomes using Fe2O3 nanoparticles. ACS Appl Nano Mater. 2021;4(2):1175-1186.

70. Yadav S, Boriachek K, Islam MN, et al. An electrochemical method for the detection of disease-specific exosomes. ChemElectroChem. 2017;4:967.

71. Palazzolo S, Memeo L, Hadla M, et al. Cancer extracellular vesicles: next-generation diagnostic and drug delivery nanotools. Cancers. 2020;12(11):3165.

72. Wahlgren J, De LKT, Brisslert M, et al. Plasma exosomes can deliver exogenous short interfering RNA to monocytes and lymphocytes. Nucleic Acids Res. 2012;40(17):e130.

73. Milman N, Ginini L, Gil Z. Exosomes and their role in tumorigenesis and anticancer drug resistance. Drug Resist Updates. 2019;45:1-12.

74. Song H, Liu B, Dong B, et al. Exosome-based delivery of natural products in cancer therapy. Front Cell Dev Biol. 2021;9:650426.

75. Saari H, Lázaro-Ibáñez E, Viitala T, Vuorimaa-Laukkanen E, Siljander P, Yliperttula M. Microvesicle- and exosome-mediated drug delivery enhances the cytotoxicity of Paclitaxel in autologous prostate cancer cells. J Controll Release. 2015;220(Pt B):727-737.

76. Jia G, Han Y, An Y, et al. NRP-1 targeted and cargo-loaded exosomes facilitate simultaneous imaging and therapy of glioma in vitro and in vivo. Biomaterials. 2018;178:302-316.

77. Osterman CJ, Lynch JC, Leaf P, et al. Curcumin modulates pancreatic adenocarcinoma cell-derived exosomal function. PLoS One. 2015;10 (7):e0132845.

78. Salarpour S, Forootanfar H, Pournamdari M, Ahmadi-Zeidabadi M, Esmaeeli M, Pardakhty A. Paclitaxel incorporated exosomes derived from glioblastoma cells: comparative study of two loading techniques. Daru. 2019;27(2):533-539.
79. Chen T, Guo J, Yang M, Zhu X, Cao X. Chemokine-containing exosomes are released from heat-stressed tumor cells via lipid raft-dependent pathway and act as efficient tumor vaccine. J Immunol. 2011;186(4):2219-2228.

80. Xie Y, Bai O, Zhang H, et al. Membrane-bound HSP70-engineered myeloma cell-derived exosomes stimulate more efficient CD8(+) CTL- and NK-mediated antitumour immunity than exosomes released from heat-shocked tumour cells expressing cytoplasmic HSP70. J Cell Mol Med. 2010;14(11):2655-2666.

81. Guo D, Chen Y, Wang S, et al. Exosomes from heat-stressed tumour cells inhibit tumour growth by converting regulatory T cells to Th17 cells via IL-6. Immunology. 2018;154(1):132-143.

82. Syn NL, Wang L, Chow EK, Lim CT, Goh BC. Exosomes in cancer nanomedicine and immunotherapy: prospects and challenges. Trends Biotechnol. 2017;35(7):665-676.

83. Xu HY, Li N, Yao N, et al. CD8+ T cells stimulated by exosomes derived from RenCa cells mediate specific immune responses through the FasL/Fas signaling pathway and, combined with GM-CSF and IL-12, enhance the anti-renal cortical adenocarcinoma effect. Oncol Rep. 2019;42(2):866-879.

84. Sharma A, Johnson A. Exosome DNA: critical regulator of tumor immunity and a diagnostic biomarker. J Cell Physiol. 2020;235 (3):1921-1932.

85. Wang Y, Ni H, Zhou S, et al. Tumor-selective blockade of CD47 signaling with a CD47/PD-L1 bispecific antibody for enhanced anti-tumor activity and limited toxicity. Cancer Immunol Immunother. 2020;70(2):365-376.

86. Xu MM, Pu Y, Han D, et al. Dendritic cells but not macrophages sense tumor mitochondrial DNA for cross-priming through signal regulatory protein $\alpha$ signaling. Immunity. 2017;47(2):363-373.e365.

87. Stuart EC, Scandlyn MJ, Rosengren RJ. Role of epigallocatechin gallate (EGCG) in the treatment of breast and prostate cancer. Life Sci. 2006;79(25):2329-2336.

88. Jang JY, Lee JK, Jeon YK, Kim CW. Exosome derived from epigallocatechin gallate treated breast cancer cells suppresses tumor growth by inhibiting tumor-associated macrophage infiltration and M2 polarization. BMC Cancer. 2013;13:421.

89. Yang MQ, Du Q, Varley PR, et al. Interferon regulatory factor 1 priming of tumour-derived exosomes enhances the antitumour immune response. Br J Cancer. 2018;118(1):62-71.

90. Dai S, Wei D, Wu Z, et al. Phase I clinical trial of autologous ascites-derived exosomes combined with GM-CSF for colorectal cancer. Mol Ther. 2008;16(4):782-790.

91. Yang Y, Xiu F, Cai Z, et al. Increased induction of antitumor response by exosomes derived from interleukin-2 gene-modified tumor cells. J Cancer Res Clin Oncol. 2007;133(6):389-399.

92. Dai S, Zhou X, Wang B, et al. Enhanced induction of dendritic cell maturation and HLA-A*0201-restricted CEA-specific CD8(+) CTL response by exosomes derived from IL-18 gene-modified CEApositive tumor cells. $J$ Mol Med. 2006;84(12):1067-1076.

93. Wang J, Wang L, Lin Z, Tao L, Chen M. More efficient induction of antitumor $\mathrm{T}$ cell immunity by exosomes from CD40L gene-modified lung tumor cells. Mol Med Rep. 2014;9(1):125-131.

94. Poggio M, Hu T, Pai CC, et al. Suppression of exosomal PD-L1 induces systemic anti-tumor immunity and memory. Cell. 2019;177 (2):414-427.e413.

95. Wang Y, Li Q, Shi H, et al. Microfluidic Raman biochip detection of exosomes: a promising tool for prostate cancer diagnosis. Lab Chip. 2020;20(24):4632-4637.

96. Xie F, Xu M, Lu J, Mao L, Wang S. The role of exosomal PD-L1 in tumor progression and immunotherapy. Mol Cancer. 2019;18(1):146. 


\section{Publish your work in this journal}

The International Journal of Nanomedicine is an international, peerreviewed journal focusing on the application of nanotechnology in diagnostics, therapeutics, and drug delivery systems throughout the biomedical field. This journal is indexed on PubMed Central, MedLine, CAS, SciSearch ${ }^{\mathbb{B}}$, Current Contents ${ }^{\mathbb{B}} /$ Clinical Medicine,
Journal Citation Reports/Science Edition, EMBase, Scopus and the Elsevier Bibliographic databases. The manuscript management system is completely online and includes a very quick and fair peer-review system, which is all easy to use. Visit http://www.dovepress.com/ testimonials.php to read real quotes from published authors. 